\title{
On enzymatic reactions: the role of a feedback from the substrate
}

\author{
Pasquale Palumbo, Mahsa Ghasemi, and Mohammad Fakhroleslam
}

\begin{abstract}
This note investigates a basic enzymatic scheme, with a substrate transforming into a product by means of the catalytic action of an enzyme. The focus is in the role of a feedback regulating the enzyme production. The novelty of the paper is in the choice of the feedback, acting from substrate accumulation differently from previous cases already studied in the literature, where the feedback acts from the product or from the enzyme. The feedback scheme is studied according to both a deterministic and stochastic approach: the former providing the existence of a unique meaningful asymptotically stable equilibrium; the latter investigating how noise propagates with or without the feedback. Regards to the stochastic approach, the metabolic noise is evaluated in terms of the coefficient of variation of the product of the enzymatic reaction, aiming at measuring its fluctuations around the average steady-state. Numerical results are carried out according to Chemical Master Equations, showing a clear improvement, in terms of noise reduction, when the negative feedback is applied. Linear Noise Approximation has been as well exploited with the aim of finding analytical solutions for the metabolic noise, relating it to the model parameters.
\end{abstract}

\section{INTRODUCTION}

Synthetic biology is a novel and challenging field of research, aiming at creating, characterizing and assembling synthetic biological circuits to be exploited in a wide range of applications, such as energy, environment and medicine [1]. In the Synthetic Biology framework, mathematical control theory has recently played a crucial role in the building of mathematical abstractions aiming at a better understanding of complex biological systems, as well as in the proposal of practical solutions, possibly taking inspiration from established engineering paradigms (see, e.g. the results recently achieved to isolate and characterise parts of engineered biological circuits, in order to understand how the different modules can be wired in more complex circuits, [2]-[4]). To this end, the role of the feedback has been widely investigated, especially in transcriptional and metabolic regulation where gene products are required to control their homeostatic levels robustly with respect to parameter or environmental fluctuations [5]-[15].

P. Palumbo (Corresponding author) is with the Istituto di Analisi dei Sistemi e Informatica, Italian National Research Research Council, Viale Manzoni, Roma, Italy. pasquale.palumbo@iasi.cnr.it

P. Palumbo is supported by the MIUR grant SysBioNet Italian Roadmap for ESFRI Research Infrastructures, SYSBIO.

M. Ghasemi is with the Department of Information Engineering, Computer Science and Mathematics, DEWS Center of Excellence, University of L'Aquila, 67100, L'Aquila, Italy.

M. Fakhroleslam is with the School of Chemical Engineering, College of Engineering, University of Tehran, P.O. Box 11365-4563, Tehran, Iran, and the Department of Information Engineering, Computer Science and Mathematics, DEWS Center of Excellence, University of L'Aquila, 67100, L'Aquila, Italy. fakhroleslameut.ac.ir
This note investigates the role of the feedback in the enzymatic production rate for a basic (though rather general) reaction scheme. Such a framework has gained a relevant interest in the recent years (e.g. [5]-[8]) with the aim of quantifying the impact of the feedback in noise propagation. Differently from the aforementioned papers, this note investigates the case of a negative feedback from the substrate, according to the scheme in Fig.1. The elementary reaction scheme refers to a substrate $S$ binding to an enzyme $E$ in order to form a complex $C$ (reaction 1) which in turn can reverse the binding (reaction 2) or can be transformed into a product $P$ (with the release of the enzyme $E$, reaction $3)$. The system under investigation accounts for enzyme production (reaction 4) and degradation (reaction 5), product elimination (reaction 6) and substrate production (reaction 7).

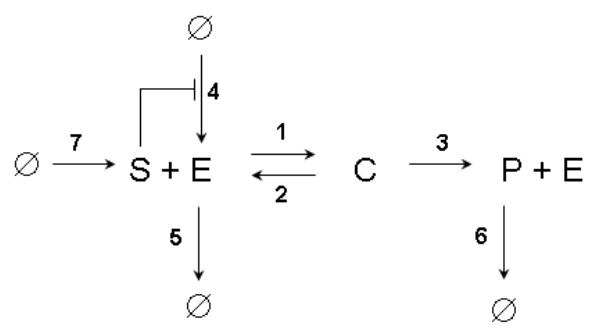

Fig. 1. Enzymatic reaction framework (Scheme 1)

The enzymatic reaction framework depicted in Fig. 1 will be addressed to as Scheme 1, with the enzyme production rate controlled in feedback by the susbtrate; on the other hand, we will refer to Scheme 0 when addressing a scheme similar to the one of Fig. 1, with the enzyme production rate not controlled in feedback by the substrate.

The feedback from the substrate provides a couple of positive steady-state equilibrium points for the deterministic Ordinary Differential Equation (ODE) system associated to the enzymatic reactions, though it is proven that only one is asymptotically stable. Such a stable equilibrium point corresponds to the steady-state solution coming from the first-order moment equations, written in closed form after standard moment closure techniques [16], [17]. As a matter of fact, Scheme 0 and Scheme 1 share the existence of a unique, positive, asymptotically stable equilibrium point.

The stochastic approach aims at quantifying the metabolic noise in terms of the product fluctuations around its steadystate average value. To this end, Chemical Master Equations (CME) are exploited [18], providing numerical solutions by means of the Gillespie Stochastic Simulation Algorithm 
TABLE I

CHEMical ReACTIONS.

\begin{tabular}{c|c|c}
\hline Event & Population reset & Propensity function \\
\hline $\begin{array}{c}\text { Substrate/enzyme } \\
\text { binding }\end{array}$ & $n_{s}(t) \mapsto n_{s}(t)-1$ & \\
& $n_{e}(t) \mapsto n_{e}(t)-1$ & $w_{1}=k_{1} n_{s}(t) n_{e}(t)$ \\
& $n_{c}(t) \mapsto n_{c}(t)+1$ & \\
\hline $\begin{array}{c}\text { Substrate/enzyme } \\
\text { unbinding }\end{array}$ & $n_{s}(t) \mapsto n_{s}(t)+1$ & \\
$n_{e}(t) \mapsto n_{e}(t)+1$ & $w_{2}=k_{2} n_{c}(t)$ \\
$n_{c}(t) \mapsto n_{c}(t)-1$ & \\
\hline $\begin{array}{c}\text { Product production/ } \\
\text { enzyme release/ }\end{array}$ & $\begin{array}{c}n_{c}(t) \mapsto n_{c}(t)-1 \\
n_{e}(t) \mapsto n_{e}(t)+1 \\
n_{p}(t) \mapsto n_{p}(t)+1\end{array}$ & $w_{3}=k_{3} n_{c}(t)$ \\
\hline $\begin{array}{c}\text { Enzyme production } \\
\text { (no feedback) }\end{array}$ & $n_{e}(t) \mapsto n_{e}(t)+1$ & $w_{4}=k_{4}$ \\
\hline $\begin{array}{c}\text { Enzyme production } \\
\text { (with feedback) }\end{array}$ & $n_{e}(t) \mapsto n_{e}(t)+1$ & $w_{4}=f\left(n_{s}(t)\right)$ \\
\hline Enzyme clearance & $n_{e}(t) \mapsto n_{e}(t)-1$ & $w_{5}=k_{5} n_{e}(t)$ \\
\hline Product clearance & $n_{p}(t) \mapsto n_{p}(t)-1$ & $w_{6}=k_{6} n_{p}(t)$ \\
\hline Substrate production & $n_{s}(t) \mapsto n_{s}(t)+1$ & $w_{7}=k_{7}$ \\
\hline \multicolumn{2}{|c}{} & \\
\hline
\end{tabular}

(SSA) [19]. To make a fair comparison between the two schemes, the feedback parameters in Scheme 1 will be set in order to provide the same equilibrium point of Scheme 0. Similarly to other cases where the feedback is exerted by the product or by the enzyme [5], [6], [7], [8], numerical results show a clear improvement, in terms of noise reduction, when the feedback is applied.

Analytical solutions of the CME would be required to refer the metabolic noise to the model parameters; unfortunately such a task is not affordable because of the curse of dimensionality that prevents to estimate the stationary distribution [18]. To cope with such a drawback different levels of approximations are here exploited, like moment equations applied after linearization of the CME propensities [16], [17] and Linear Noise Approximation (LNA) [18]. Both cases allow to compute the second-order moments by solving a 10th order linear system of algebraic equations and do not allow to have an easy-to-handle analytical form for the metabolic noise. Therefore, on top of LNA, the Quasi-Steady-State Approximation (QSSA) is applied [20], [21], which properly exploits the double time-scale of the system, typical of enzymatic reactions frameworks, to reduce the complexity of the slow dynamics. The correctness of both approximations is validated by the comparison with the numerical solutions provided by the SSA.

\section{MATHEMATICAL MODEL OF THE CHEMiCAL REACTION SCHEME}

Let $\left[\begin{array}{llll}n_{s} & n_{e} & n_{c} & n_{p}\end{array}\right]^{T}$ denote the copy number of substrate, enzyme, complex and product, respectively. According to the scheme of Fig. 1 the model accounts for 7 reactions, $\left\{r_{1}, \ldots, r_{7}\right\}$, each modeled by a random event occurring probabilistically at exponentially distributed time instants, with the temporal evolution of the reaction network described by a Continuous-Time Markov Process, where a state-dependent propensity $w_{j}$ is associated to reaction $j$, [18]. The details of the chemical reactions are resumed in the Table I including the resets on the players and the propensities associated to each reaction.

Regards to Scheme 1, we model the propensity $w_{4}$ by means of the following Hill function:

$$
w_{4}\left(n_{s}\right)=f\left(n_{s}\right)=\frac{\beta}{1+\left(n_{s} / \theta\right)^{M}}
$$

Parameter $\beta$ provides the maximal propensity (the promoter strength), obtainable for negligible values of its entry $n_{s}$. Half of the maximal value of the propensity is reached in correspondence of the repression thresholds, $\theta$. Parameter $M$ is the promoter sensitivity, providing the steepness of the sigmoidal function. Regards to Scheme 0 , the propensity $w_{4}$ is trivially given by a constant parameter $k_{4}$.

\section{A. Metabolic noise}

Similarly to [5], [6], [7], [8], we define the metabolic noise related to the product $P$ in terms of the coefficient of variation:

$$
C V_{p}^{2}=\frac{\sigma_{p}^{2}}{\left(n_{p}^{\star}\right)^{2}}
$$

where $\sigma_{p}^{2}$ and $n_{p}^{\star}$ are the steady-state values for variance and average of the marginal distribution of the product $P$ 's copy number.

The comparison between the two schemes (with or without feedback) is carried out by properly setting the feedback parameters in order that the same steady-state average values are provided. As a matter of fact, $n_{p}^{\star}$ does not vary in the two schemes (actually $n_{p}^{\star}$ does not vary independently of the feedback parameters, as it will be clearer in the next Section).

In the following, the expected value (average value) of a random variable $x$ is denoted by $\langle x\rangle$, while the steady-state of the expected value of a stochastic process $x(t)$ is denoted by $x^{\star}=\lim _{t \mapsto+\infty}\langle x(t)\rangle$.

\section{FIRST-ORDER MOMENTS}

Consider the scheme with the feedback. By properly exploiting the mass-action law, the following ODE system can be associated to the set of chemical players, where the state variables $\left[\left\langle n_{s}\right\rangle\left\langle n_{e}\right\rangle\left\langle n_{c}\right\rangle\left\langle n_{p}\right\rangle\right]^{T}$ indicate the average values.

$$
\begin{aligned}
& \frac{d\left\langle n_{s}\right\rangle}{d t}=-k_{1}\left\langle n_{s}\right\rangle\left\langle n_{e}\right\rangle+k_{2}\left\langle n_{c}\right\rangle+k_{7}=0 \\
& \frac{d\left\langle n_{e}\right\rangle}{d t}=-k_{1}\left\langle n_{s}\right\rangle\left\langle n_{e}\right\rangle+\left(k_{2}+k_{3}\right)\left\langle n_{c}\right\rangle-k_{5}\left\langle n_{e}\right\rangle \\
& +f\left(\left\langle n_{s}\right\rangle\right)=0 \\
& \frac{d\left\langle n_{c}\right\rangle}{d t}=k_{1}\left\langle n_{s}\right\rangle\left\langle n_{e}\right\rangle-\left(k_{2}+k_{3}\right)\left\langle n_{c}\right\rangle=0 \\
& \frac{d\left\langle n_{p}\right\rangle}{d t}=k_{3}\left\langle n_{c}\right\rangle-k_{6}\left\langle n_{p}\right\rangle=0
\end{aligned}
$$

Due to the nonlinear propensities, the ODE system is an approximation coming from the first-order moment equations, whose higher order terms are approximated by firstorder moments, by means of moment closure techniques (see e.g. [17]).

According to this setting, steady-state solutions for complex and product are easily achieved as

$$
n_{c}^{\star}=\frac{k_{7}}{k_{3}}, \quad n_{p}^{\star}=\frac{k_{7}}{k_{6}} .
$$


Instead, steady-state solution for substrate has to be numerically searched from the roots of the following polynomial:

$\varphi\left(n_{s}^{\star}\right)=K\left(n_{s}^{\star}\right)^{M}-\theta^{M} n_{s}^{\star}+K \theta^{M}, \quad K=\frac{k_{5} k_{7}\left(k_{2}+k_{3}\right)}{k_{1} k_{3} \beta}$,

with steady-state solution for the enzyme coming straightforwardly from

$$
n_{e}^{\star}=\frac{\beta}{k_{5}} \cdot \frac{1}{1+\left(n_{s}^{\star} / \theta\right)^{M}} .
$$

Theorem 1: The ODE system (3) admits a pair of positive real equilibrium points if, and only if, the following inequality is satisfied.

$$
\theta>K M(M-1)^{-\frac{M-1}{M}} .
$$

Otherwise, no positive real equilibrium points occur.

Proof: The problem of finding positive real equilibrium points is that of finding positive real solutions to $\varphi\left(n_{s}^{\star}\right)=0$ in (5). To this end consider the derivative

$$
\begin{aligned}
& \varphi^{\prime}\left(n_{s}^{\star}\right)=K M\left(n_{s}^{\star}\right)^{M-1}-\theta^{M}>0 \\
& \Longrightarrow \quad n_{s}^{\star}>n_{s, \text { min }}=\left(\frac{\theta^{M}}{K M}\right)^{\frac{1}{M-1}}
\end{aligned}
$$

stating the existence of a unique positive minimum at $n_{s, \min }$. Since $\varphi(0)=K \theta^{M}>0$, then there can be only 2 scenarios: one that prevents real roots, provided that $\varphi\left(n_{s, \text { min }}\right)>0$, and the other providing 2 real positive roots, provided that $\varphi\left(n_{s, \min }\right)<0$. This last condition is shown to provide condition (7) after some computations.

In order to investigate the stability of the equilibrium points (occurring when condition (7) is satisfied), we consider the Jacobian $J$ associated to (3) providing the following characteristic polynomial:

$$
d(\lambda)=\operatorname{det}(\lambda I-J)=\lambda^{3}+a_{2} \lambda^{2}+a_{1} \lambda+a_{0},
$$

where

$$
\begin{aligned}
& a_{2}=k_{1}\left(n_{s}^{\star}+n_{e}^{\star}\right)+k_{2}+k_{3}+k_{5} \\
& a_{1}=k_{1}\left(k_{5} n_{e}^{\star}+n_{s}^{\star} f^{\prime}\left(n_{s}^{\star}\right)\right)+k_{1} k_{3} n_{e}^{\star}+k_{5}\left(k_{2}+k_{3}\right) \\
& a_{0}=k_{1} k_{3}\left(k_{5} n_{e}^{\star}+n_{s}^{\star} f^{\prime}\left(n_{s}^{\star}\right)\right)
\end{aligned}
$$

By applying the Routh criterion, $d(\lambda)$ admits roots with negative real part (thus ensuring asymptotic stability) if, and only if

$$
a_{0}>0 \quad \text { and } \quad a_{1} a_{2}-a_{0}>0 .
$$

However, the second of (11) becomes

$a_{1} a_{2}-a_{0}=a_{2}\left(k_{1} k_{3} n_{e}^{\star}+k_{5}\left(k_{2}+k_{3}\right)\right)+a_{0}\left(\frac{a_{2}}{k_{3}}-1\right)>0$.

After computations, it can be shown that

$$
\frac{a_{2}}{k_{3}}-1=\frac{k_{1}\left(n_{s}^{\star}+n_{e}^{\star}\right)+k_{2}+k_{5}}{k_{3}}>0,
$$

therefore

$$
a_{0}>0 \quad \Longrightarrow \quad a_{1} a_{2}-a_{0}>0,
$$

with condition

$$
a_{0}>0 \quad \Longleftrightarrow \quad n_{s}^{\star}<\tilde{n}_{s}=\theta(M-1)^{-\frac{1}{M}} .
$$

achieved by properly exploiting the steady-state condition (6) and the derivative of $f(\cdot)$.

Remark 2: It is worthwhile to notice that, differently from (7), condition (15) requires to be numerically verified for both the equilibrium points, since it involves the computation of the substrate steady-state. The following theorem allows to overcome such a drawback, and simplifies the investigation of steady-state stability.

Theorem 3: Assume that condition (7) is satisfied, so that system (3) admits a pair of positive real steady-state solutions. Then, the steady-state associated to the smaller value of $n_{s}^{\star}$ is asymptotically stable, whilst the one associated to the greater value of $n_{s}^{\star}$ is unstable.

Proof: Denote with $0<n_{s, 1}^{\star}<n_{s, 2}^{\star}$ the pair of positive real equilibrium points. According to (5), the pair of solutions are given by the intersections of a Hill function with a hyperbola as

$$
\frac{1}{1+\left(n_{s, i}^{\star} / \theta\right)^{M}}=\frac{K}{n_{s, i}^{\star}}, \quad i=1,2,
$$

with the positive $n_{s}^{\star}$-semi-axis that can be partitioned in three regions of

$$
[0,+\infty)=\left[0, n_{s, 1}^{\star}\right) \cup\left[n_{s, 1}^{\star}, n_{s, 2}^{\star}\right) \cup\left[n_{s, 2}^{\star},+\infty\right)
$$

In the first and third regions the hyperbola is over the Hill function; the opposite occurs in the intermediate region. Now, consider $\tilde{n}_{s}$ defined in (15) and assume that $\tilde{n}_{s} \in\left[n_{s, 1}^{\star}, n_{s, 2}^{\star}\right.$ ), that means:

$$
\frac{1}{1+\left(\tilde{n}_{s} / \theta\right)^{M}}>\frac{K}{\tilde{n}_{s}} \text {. }
$$

If condition (18) is satisfied, then

$$
n_{s, 1}^{\star}<\tilde{n}_{s}<n_{s, 2}^{\star},
$$

from which, by virtue of (15), the smaller $n_{s, 1}^{\star}$ is asymptotically stable, whilst the larger $n_{s, 2}^{\star}$ is unstable.

Inequality (18) becomes (7) by trivially substituting $\tilde{n}_{s}$.

In summary, provided condition (7) is satisfied, there exists a unique real positive, asymptotically stable equilibrium point, provided by (4), (6) and the smaller of the positive real solutions of $\varphi\left(n_{s}^{\star}\right)=0$ in (5). Therefore, the presence of the feedback modifies the values of $n_{s}^{\star}$ and $n_{e}^{\star}$, but not the ones related to $n_{c}^{\star}$ and $n_{p}^{\star}$. In order to make fair comparisons between the two schemes (with or without feedback), similarly to [5], the feedback parameters will be tuned in order to provide the same average steady-state solution for the non-feedback case, namely

$$
n_{c}^{\star}=\frac{k_{7}}{k_{3}}, \quad n_{p}^{\star}=\frac{k_{7}}{k_{6}}, \quad n_{e}^{\star}=\frac{k_{4}}{k_{5}}, \quad n_{s}^{\star}=\frac{k_{5} k_{7}\left(k_{2}+k_{3}\right)}{k_{1} k_{3} k_{4}} .
$$

For instance, imaging to fix all the other parameters, as well as the feedback sensitivity $M$, the pair $(\beta, \theta)$ must satisfy the identity

$$
k_{4}=\frac{\beta}{1+\left(n_{s}^{\star} / \theta\right)^{M}}
$$


TABLE II

THE PARAMETERS OF THE ENZYMATIC MODEL.

\begin{tabular}{c|c|c}
\hline Parameter & Value & Unit \\
\hline$k_{1}$ & 1 & $\mathrm{~s}^{-1}$ molecule $^{-1}$ \\
$k_{2}$ & 28 & $\mathrm{~s}^{-1}$ \\
$k_{3}$ & 3.2 & $\mathrm{~s}^{-1}$ \\
$k_{4}$ & 0.16 & molecule/s \\
$k_{5}$ & 0.02 & $\mathrm{~s}^{-1}$ \\
$k_{6}$ & 0.1 & $\mathrm{~s}^{-1}$ \\
$k_{7}$ & 2.4 & molecule/s \\
\hline
\end{tabular}

with $n_{s}^{\star}$ as in (20). Fig. 2 shows some of these curves, for different values of the promoter sensitivities $M$. Other model parameters are given in Table II.

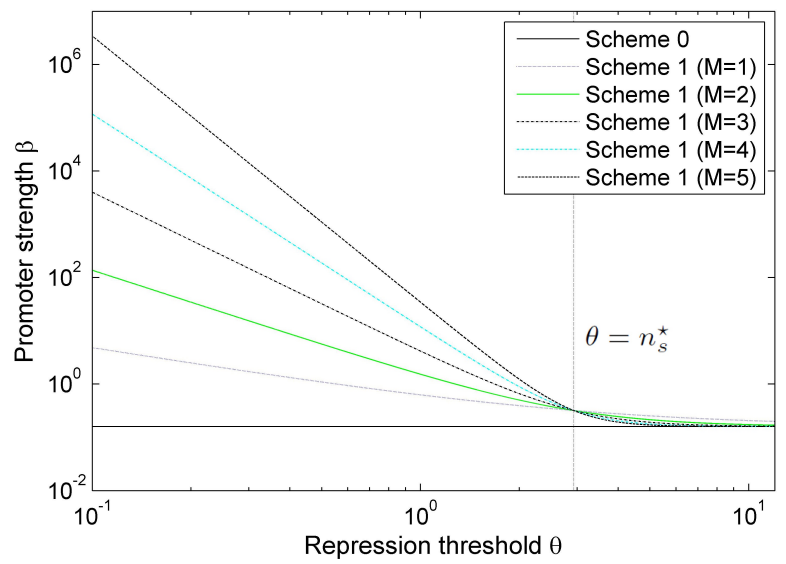

Fig. 2. ( $\beta$ vs $\theta)$-curves ensuring fixed values of $n_{s}^{\star}$ for different values of the promoter sensitivity $M$. The vertical dashed line depicts $\theta=n_{s}^{\star}$.

Remark 4: As it can be seen from Fig. 2, given $n_{s}^{\star}$ from Scheme 0 , all $(\beta, \theta)$-curves satisfying (21) pass through the point $\left(2 k_{4}, n_{s}^{\star}\right)$ for any promoter sensitivity $M$. Moreover, by increasing the value of the threshold $\theta \gg n_{s}^{\star}$, the effect of the feedback becomes weaker and the promoter strength $\beta$ approaches $k_{4}$ : a feedback with a threshold very large with respect to the steady-state $n_{s}^{\star}$ requires the minimum value for its strength to provide the same steady-state $n_{e}^{\star}$ as without feedback, see (6). Instead, by decreasing the value of the threshold $\theta \ll n_{s}^{\star}$, the effect of the feedback becomes stronger and the promoter strength $\beta$ should be increased to have the same enzyme stationary mean value, see (6).

\section{Computation of THE MEtabolic nOISE}

The proper way to compute the metabolic noise defined in (2) is by means of the steady-state probability distribution provided by the solution of the CME associated to the set of chemical reactions. However, to find such a solution for a CME is a hard nut to crack because of the curse of dimensionality which, unless simple cases involving few copy numbers of the involved species, prevents from explicitly computing the stationary distribution, in favor of efficient algorithms [22], [23] or Monte Carlo methods (e.g. the Gillespie SSA [19]) providing affordable estimates of the CME solution. For these reasons, CME solutions are here provided numerically from SSA. However, in the case of enzymatic reactions, model parameters use to be set in order to show a double time-scale of the reactions involved (reactions of binding/unbinding occur on a shorter timescale than the other reactions), and this makes exact SSA computationally demanding, since it gets stuck on thousands of binding/unbinding reactions for each birth of a product molecule [8]. In this note, model parameters (see Table II) have been modified from usual data coming from literature [5], [6], [7], [8] in order to weaken such a double-time scale property, and allow affordable results from the standard SSA: as a matter of fact, the present scheme should be considered as a toy model providing qualitative suggestions for further research based on a more meaningful parameter setting.

Simulation results are reported in Fig. 3, where the metabolic noise is pictured for the cases with and without feedback according to different values of the threshold $\theta$ and of the feedback sensitivity $M$. The SSA results are denoted by circles and refer only to the feedback case (Scheme 1). As previously stated, they are achieved by arbitrarily varying $\theta$ and then constraining $\beta$ to $\theta$ according to (21).

\section{A. Analytical solution by means of 2 nd order moment equa- tions}

Instead of looking for the whole stationary distribution (and, then, provide from it the first and second order moments required to compute the metabolic noise), one may think at achieving only first and second order moments. However, these moment equations are not in closed form, because of the nonlinear propensities $w_{1}$ and $w_{4}$, this last in the feedback case (see Table I). To avoid moment closure techniques [16], [17], similarly to [10] under the hypothesis of small noise fluctuations around the average steady state copy numbers, the nonlinear propensities are linearized by means of the first order approximation, which enables to obtain closed moment equations:

$$
\begin{aligned}
k_{1} n_{s}(t) n_{e}(t) & \simeq k_{1}\left(n_{s}^{\star} n_{e}^{\star}+n_{e}^{\star}\left(n_{s}(t)-n_{s}^{\star}\right)+n_{s}^{\star}\left(n_{e}(t)-n_{e}^{\star}\right)\right) \\
f\left(n_{s}(t)\right) & \simeq f\left(n_{s}^{\star}\right)+f^{\prime}\left(n_{s}^{\star}\right)\left(n_{s}(t)-n_{s}^{\star}\right)
\end{aligned}
$$

The second order moment equations now provide a linear 10 -th order algebraic system with respect to the unknown

$$
\left\langle n_{i} n_{j}\right\rangle, \quad i, j=s, e, c, p .
$$

From $\left\langle n_{p}^{2}\right\rangle^{\star}$ and $p_{n}^{\star}$ the metabolic noise is then computed for Scheme 1 and is reported in Fig. 3 under the label 2nd order moment, according to different setting of the threshold $\theta$. If compared to the corresponding metabolic noise of the non feedback case (Scheme 0), it is apparent the improvement in terms of noise reduction.

The use of the adopted approximation for the nonlinear propensities is validated by the SSA simulations, that appear to be close to the approximated ones (besides the fact that the two methods share the same qualitative behavior for varying $\theta$ ). Differently to the SSA case, these solutions can be achieved in a faster way and provide richer sets of simulated data but, unfortunately, they do not provide an easy-to-handle analytical formula for $C V_{p}^{2}$. To this end, a different approximation is exploited in the next Section. 


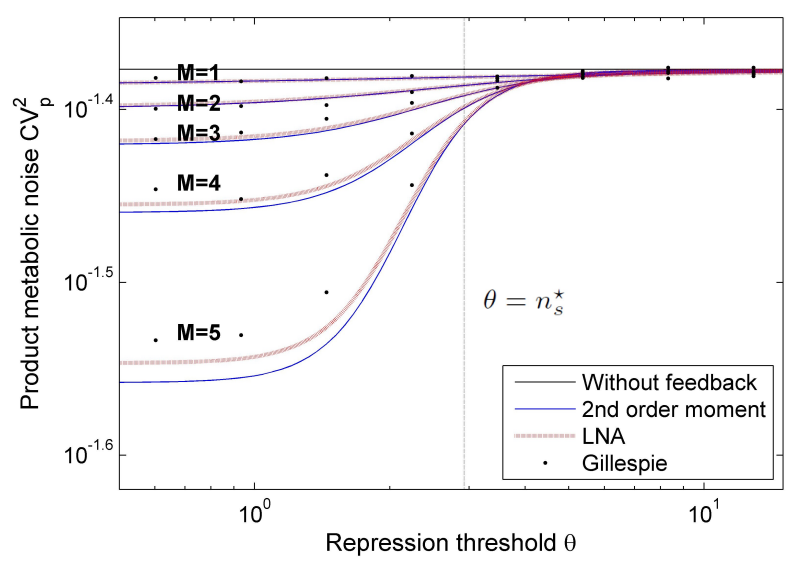

Fig. 3. The metabolic noise for different values of $M$ and $\theta$. Comparison of the results without feedback and with feedback obtained from the solution of the second order moment equations, LNA framework, and Gillespie simulations. The vertical dashed line shows $\theta=n_{s}^{\star}$.

\section{B. Analytical solution by means of LNA}

The LNA provides the stationary distribution of the copy numbers in terms of a multivariate Gaussian, according to the simplifying assumption that molecular fluctuations produce small deviations around the stationary average values. Mean values of the LNA distribution are computed from the steadystate solution of the deterministic ODE system associated to the chemical reactions (3), written with respect to the concentrations (instead of copy numbers). The covariance matrix comes out as the positive definite solution of a properly stated Lyapunov equation, written according to the linearization of the ODE.

From a computational viewpoint, LNA provides secondorder moments as the solution of a linear 10th order algebraic system (the unknowns are the 10 independent entries of the covariance matrix in the Lyapunov equation). These solutions are, in general, different from the ones achieved by moment equations discussed in Section IV-A although they share the same computational burden. However, similarly to [8], [7], the Quasi Steady-State Approximation (QSSA) can be applied on top of LNA to further reduce computations. The QSSA is widely exploited to lighten the computational complexity in presence of the typical fast/slow time-scale of enzymatic reactions [20], [21]. It substantially neglects the highly reactive species (in this system, complex $C$ ), whose dynamics is supposed to be over with respect to the others: as a matter of fact the complex $C$ dynamic equation is substituted by the algebraic equation coming from $d n_{c} / d t=0$. This approximation clearly does not affect the steady-states, instead it allows to simplify the corresponding Lyapunov equation from a 10th order to a 6th order system.

To apply the LNA+QSSA, define $\gamma=\left[\begin{array}{llll}\gamma_{s} & \gamma_{e} & \gamma_{c} & \gamma_{p}\end{array}\right]^{T}$ as the vector of concentrations associated to the vector of copy numbers $\left[n_{s} n_{e} n_{c} n_{p}\right]^{T}$ in a constant distribution volume $V$. Then, exploit the algebraic equation provided by $d \gamma_{c} / d t=0$ to compute $\gamma_{c}$ as a function of other concentrations, and substitute it into the other dynamic equations. The new 3rd order ODE system can be restated in the following more compact form with respect to a new definition of

$$
\begin{array}{ll}
\gamma=\left[\begin{array}{lll}
\gamma_{s} & \gamma_{e} & \gamma_{p}
\end{array}\right]^{T}: & \\
\dot{\gamma}(t)=N \mathcal{V}(\gamma(t))
\end{array}
$$

where $N$ is the stoichiometric matrix

$$
N=\left[\begin{array}{ccccc}
-1 & 0 & 0 & 0 & 1 \\
0 & 1 & -1 & 0 & 0 \\
1 & 0 & 0 & -1 & 0
\end{array}\right]
$$

associated to vector $\mathcal{V}$ collecting the macroscopic reaction rates:

$$
\mathcal{V}=\left[\frac{k_{1} k_{3}}{k_{2}+k_{3}} \gamma_{s} \gamma_{e} V, \frac{\delta\left(\gamma_{s}\right)}{V}, k_{5} \gamma_{e}, k_{6} \gamma_{p}, \frac{k_{7}}{V}\right]^{T}
$$

where $\delta\left(\gamma_{s}\right)=k_{4}$ for the case without feedback (Scheme 0), and $\delta\left(\gamma_{s}\right)=f\left(\gamma_{s} V\right)$ for the case with the feedback (Scheme 1).

The covariance matrix $\Sigma$ associated to the copy number fluctuations around the equilibrium solution of (24) is the positive definite solution of the Lyapunov equation

$$
J \Sigma+\Sigma J+V B B^{T}=0
$$

with

$$
J=\left.N \cdot \frac{d \mathcal{V}}{d \gamma}\right|_{\gamma^{\star}}=\left[\begin{array}{ccc}
-\frac{k_{1} k_{3}}{\left(k_{2}+k_{3}\right)} \gamma_{e}^{\star} V & -\frac{k_{1} k_{3}}{\left(k_{2}+k_{3}\right)} \gamma_{s}^{\star} V & 0 \\
\delta^{\prime}\left(\gamma_{s}\right) & -k_{5} & 0 \\
\frac{k_{1} k_{3}}{\left(k_{2}+k_{3}\right)} \gamma_{e}^{\star} V & \frac{k_{1} k_{3}}{\left(k_{2}+k_{3}\right)} \gamma_{s}^{\star} V & -k_{6}
\end{array}\right]
$$

and

$$
B B^{T}=N \operatorname{diag}\left(\mathcal{V}\left(\gamma^{\star}\right)\right) N^{T}=\left[\begin{array}{ccc}
\frac{2 k_{7}}{V} & 0 & -\frac{k_{7}}{V} \\
0 & 2 k_{5} \gamma_{e}^{\star} & 0 \\
-\frac{k_{7}}{V} & 0 & \frac{2 k_{7}}{V}
\end{array}\right] .
$$

By solving the Lyapunov equation, we find for Scheme 0 (without feedback) and Scheme 1 (with feedback) the following analytic expression for the variance of the product, $\sigma_{p, 0}^{2}$ and $\sigma_{p, 1}^{2}$ respectively:

$$
\begin{aligned}
& \sigma_{p, 0}^{2}=\frac{k_{7}}{k_{6}}+\frac{k_{5} k_{7}^{2}}{\Xi_{0}\left(n_{e}^{\star}\right)\left(k_{6}+\frac{k_{1} k_{3} n_{e}^{\star}}{k_{2}+k_{3}}\right)}, \\
& \sigma_{p, 1}^{2}=\frac{k_{7}}{k_{6}}+\frac{k_{5} k_{7}^{2}(1-\mathcal{A})}{\Xi_{1}\left(n_{e}^{\star}\right)\left(k_{6}+\frac{k_{1} k_{3} n_{e}^{\star}}{k_{2}+k_{3}}\right)},
\end{aligned}
$$

where

$$
\begin{gathered}
\Xi_{0}\left(n_{e}^{\star}\right)=n_{e}^{\star}\left(k_{5}+\frac{k_{1} k_{3} n_{e}^{\star}}{k_{2}+k_{3}}\right)\left(k_{5}+k_{6}\right), \\
\Xi_{1}\left(n_{e}^{\star}\right)=\Xi_{0}\left(n_{e}^{\star}\right)(1-\mathcal{B})
\end{gathered}
$$

and $\mathcal{A}, \mathcal{B}>0$ are defined by:

$$
\mathcal{A}=-\frac{f^{\prime}\left(n_{s}^{\star}\right)}{k_{5}}, \quad \mathcal{B}=-\frac{k_{7} f^{\prime}\left(n_{s}^{\star}\right)}{n_{e}^{\star}\left(k_{6}+\frac{k_{1} k_{3} n_{e}^{\star}}{k_{2}+k_{3}}\right)\left(k_{5}+k_{6}\right)} .
$$

Fig. 3 shows the results obtained by LNA+QSSA in comparison to the results of the solution of the 2nd-order moment equation and the SSA. As it can be seen, the LNA+QSSA 
solutions agree with the 2 nd order moments and with the samples from the numerical Gillespie SSA. As an overall comment on Fig. 3, according to the chosen set of model parameters (see Table II), the results show the effectiveness of the negative feedback from the substrate in reducing the random fluctuations. Fig. 3 shows that by increasing the repression threshold $\theta$, the reduction of metabolic noise attenuates, definitely approaching no reduction at all for $\theta \mapsto+\infty$. Indeed, by increasing $\theta$ for values of $\theta \gg n_{s}^{\star}$, the feedback function approximates with $\beta=k_{4}$, in order to ensure the same steady-states, and Scheme 1 collapses to Scheme 0 . On the other hand, when $\theta$ reduces so that $\theta \ll n_{s}^{\star}$ then the Hill function provides a stronger attenuation of $\beta$, that is required to dramatically increase (according to Fig. 2) in order to keep fixed the steady-states. In this case, numerical simulations suggest a plateau for $\theta \mapsto 0^{+}$. Indeed, such a plateau can be computed from analytical solutions (30)-(34) since, taking into account also (21):

$$
\lim _{\theta \mapsto 0^{+}} f^{\prime}\left(n_{s}^{\star}\right)=-\frac{M k_{4}}{n_{s}^{\star}}
$$

and this value can substituted in $\mathcal{A}$ and $\mathcal{B}$ providing the limit point (that depends of the feedback sensitivity $M$ ) for $\sigma_{p, 1}^{2}$ as $\theta \mapsto 0^{+}$.

To better appreciate the improvements in noise reduction provided by the substrate feedback, Fig. 4 shows the variations of the ratio $\frac{C V_{p, 1}^{2}}{C V_{p, 0}^{2}}$ with different parameters $M$ by varying the threshold $\theta$ of the feedback function.

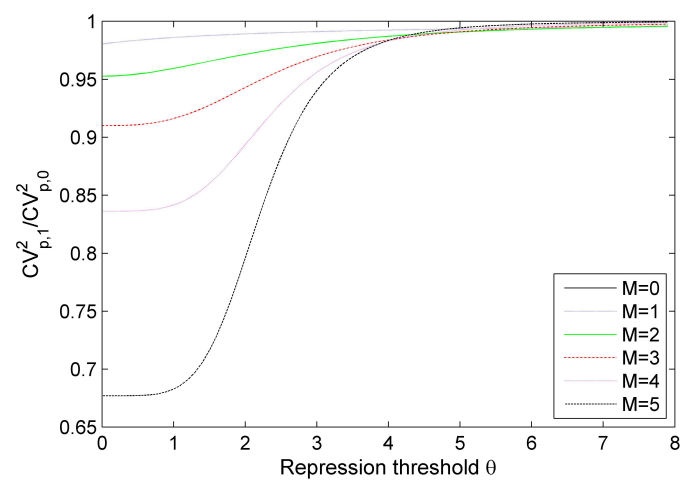

Fig. 4. The ratio of the metabolic noises $C V_{p, 1}^{2}$ over $C V_{p, 0}^{2}$ for different values of the feedback parameters $M$ and $\theta$.

\section{Conclusions}

The role of a negative feedback from the substrate in a basic enzymatic scheme has been investigated, with respect to the occurrence of asymptotically stable equilibrium points and noise propagation. Noise propagation has been studied according to different stochastic approaches. From one hand, the general framework of Chemical Master Equations has been exploited to run the Gillespie Stochastic Simulation Algorithm providing numerical solutions for a chosen set of model parameters. From the other hand, analytical results have been searched according to linear approximations of the original nonlinear propensities. To this end, second order moment equations and Linear Noise + Quasi Steady-State Approximations have been reported, these last providing an easy-to-handle analytical solution for the metabolic noise. Results achieved by approximation agree with the numerical ones provided by SSA and show a decisive role in the negative feedback to attenuate noise.

\section{ACKNOWLEDGEMENTS}

PP is supported by the MIUR grant SysBioNet Italian Roadmap for ESFRI Research Infrastructures, SYSBIO Centre of Systems Biology, Milan and Rome, Italy.

\section{REFERENCES}

[1] D. Del Vecchio, A.J. Dy, Y. Qian, Control theory meets synthetic biology, J. R. Soc. Interface, 13: 20160380. http://dx.doi.org/10.1098/rsif.2016.0380

[2] D. Del Vecchio, and E. D. Sontag, Synthetic biology: A systems engineering perspective, "Control Theory and Systems Biology", P.A. Iglesias \& B.P. Ingalls Eds., The MIT Press, 101-124, 2009.

[3] D. Del Vecchio, A. J. Ninfa, and E. D. Sontag, Modular cell biology: retroactivity and insulation, Molecular systems biology, 4.1, 161, 2008.

[4] D. Del Vecchio, Modularity, context-dependence, and insulation in engineering biological circuits, Trends in Biotechnology, 33(2), 111119, 2015.

[5] A. Borri, P. Palumbo, A. Singh, Impact of negative feedback in metabolic noise propagation, IET Syst. Biol., 1-8, 2016.

[6] A. Borri, P. Palumbo, A. Singh, Noise reduction for enzymatic reactions: a case study for stochastic product clearance, Proc. 55th IEEE Conf. Dec. Contr., Las Vegas, USA, 5851-5856, 2016.

[7] A. Borri, P. Palumbo, A. Singh, Metabolic noise reduction for enzymatic reactions: the role of a negative feedback, Proc. 54th IEEE Conf. Dec. Contr., Osaka, Japan, 2537-2542, 2015.

[8] Oyarzun, D.A, Lugagne, J.-B., Stan, G.-B.V.: Noise propagation in synthetic gene circuits for metabolic control, ACS Synth. Biol., 116$125,2014$.

[9] W.J. Holtz, J.D. Keasling, Engineering static and dynamic control of synthetic pathways, Cell, 140, 19-23, 2010.

[10] A. Singh, J.P. Hespanha, Optimal feedback strength for noise suppression in autoregulatory gene networks, Biophys. J. 96, 4013-23, 2009.

[11] U. Alon, An Introduction to Systems Biology: Design Principles of Biological Circuits, Chapman and Hall/CRC, 2006.

[12] E.D. Sontag, Some new directions in control theory inspired by systems biology, Syst. Biol. 1(1), 9-18, 2004.

[13] A. Zaslaver, A. Mayo, R. Rosenberg, P. Bashkin, H. Sberro, M. Tsalyuk, M. Surette, U. Alon, Just-in-time transcription program in metabolic pathways, Nat. Genet. 36, 486-491, 2004.

[14] G. Stephanopoulos, A. Aristidou, J. Nielsen, Metabolic Engineering: Principles and Methodologies, Academic Press, San Diego, CA, 1998.

[15] D.H. Calhoun, G.W. Hatfield, Autoregulation: a role for a biosynthetic enzyme in the control of gene expression, Proc. Natl. Acad. Sci. USA, 70(10), 2757-2761, 1973.

[16] J.P. Hespanha, A. Singh, Stochastic models for chemically reacting systems using polynomial stochastic hybrid systems, Int. J. of Robust and Nonlinear Control 15, 669-689, 2005.

[17] A. Singh, J.P. Hespanha, Approximate moment dynamics for chemically reacting systems, IEEE Trans. Autom. Control 56, 414-418, 2011.

[18] N.G. van Kampen, Stochastic Processes in Physics and Chemistry, North Holland, third edition, 2007.

[19] D. T. Gillespie, Exact Stochastic Simulation of Coupled Chemical Reactions, The Journal of Physical Chemistry 81(25), 23402361, 1977.

[20] Segel, L.: On the validity of the steady state assumption of enzyme kinetics. Bull. Math. Biol. 50, 579593, 1988

[21] A.M. Bersani, E. Bersani, L. Mastroeni, Deterministic and stochastic models of enzymatic networksapplications to pharmaceutical research, Comput. Math. Appl., 55, 879-888, 2008.

[22] A. Borri, F. Carravetta, G. Mavelli, P. Palumbo, Some Results on the Structural Properties and the Solution of the Chemical Master Equation, Proc. Amer. Contr. Conf., Washington, DC, 17-19, 2013.

[23] P.F. Tupper, Ergodicity and the numerical simulation of Hamiltonian systems, SIAM J. Appl. Dyn. Syst., 4(3), 563-587, 2005. 